\title{
Entrevista semiestructurada para la exploración de la violencia sexual sobre las mujeres en la relación de la pareja (EVS).
}

Fecha de recepción: 23/03/2016

Fecha de aceptación: 02/06/2016

\author{
Alicia Martínez Sanz \\ Departamento de Psicología Evolutiva y Didáctica. Facultad de \\ Educación. Universidad de Alicante. \\ Carmen Mañas Viejo \\ Departamento de Psicología Evolutiva y Didáctica. Facultad de \\ Educación. Universidad de Alicante. \\ Gemma Pons Salvador \\ Departamento de Psicología Básica. Facultad de Psicología. \\ Universidad de Valencia.
}

\section{resumen/ahstract:}

La violencia sexual en el ámbito de la relación de pareja es un problema grave experimentado por un alto número de mujeres. Sin embargo, es difícil su detección por la ausencia de reconocimiento social. Este tipo de violencia, con frecuencia, se da ligado a otras formas de maltrato. No obstante observamos que diferentes protocolos de entrevista publicados para explorarla, no la evalúan o lo hacen de forma superficial. Esto se suma al hecho de que algunas de las mujeres que lo sufren no son conscientes del maltrato, ni de la gravedad e impacto real que tiene sobre ellas. Por todo ello, nuestro objetivo es proponer una herramienta de recogida de información sobre la violencia sexual en la pareja, que facilite a las y los profesionales la detección de esta forma de violencia. Para ello, nos hemos basado en una revisión exhaustiva de la literatura científica y profesional y en nuestra propia experiencia. Como resultado presentamos la Entrevista Semiestructurada para la Exploración de la Violencia Sexual sobre las Mujeres en la Relación de Pareja (EVS), esperando que sea de utilidad a las y los profesionales que intervienen con mujeres que son víctimas de malos tratos.

Sexual violence in intimate partner context is a serious problem that is experienced by a large number of women. However, its detection is difficult becasuse the absence of social recognition. This kind of violence occurs frequently linked to other abuse types, however we realize that various interview protocols that have been published to explore it, don't examine it or examine it superficially. In addition, some of women who are abused, are not aware of this, neither its gravity nor real impact on them. Therefore, our purpose is to create a tool for collecting on information about intimate partner sexual violence and to help professionals in detecting this kind of violence. So, we have relied on a comprehensive review of the scientific and professional literature and our own experience. As a result we present the Entrevista Semiestructurada para la Exploración de la Violencia Sexual sobre las Mujeres en la Relación de Pareja (EVS), we hope to be useful for professionals who work with women who are victims of this abuse.

\section{palabras clave/keywords:}

Entrevista semiestructurada, violencia sexual en la pareja, violencia contra las mujeres, violencia de género, detección y exploración de la violencia sexual.

Semistructured interview, intimate partner sexual violence, violence against women, gender violence, detection and exploration of sexual violence. 


\section{dossier}

\section{Introducción}

La necesidad de crear la Entrevista Semiestructurada para la Exploración de la Violencia Sexual sobre las Mujeres en la Relación de Pareja (EVS), surgió a partir de la experiencia profesional en servicios de atención psicológica especializados en mujeres víctimas de violencia de género ${ }^{1}$. El primer contacto presencial de las mujeres con los servicios de atención especializados consiste en una entrevista en la que las mujeres relatan su historia y se recogen sus demandas explícitas e implícitas. En función de estas demandas y de la valoración profesional se les orienta al recurso jurídico, social y/o psicológico. En el servicio de atención psicológica, se realiza una valoración diagnóstica, y es en esta fase de la valoración donde se detectó la necesidad de explorar la violencia sexual de manera rigurosa, de la misma forma que se suele hacer con la violencia física y la psicológica. Cuando se preguntaba a las mujeres si habían sido forzadas a mantener relaciones sexuales por sus parejas, muchas contestaban negativamente en un primer momento. Sin embargo, cuando se les planteaba si se habían visto obligadas, de alguna manera, a tener relaciones sexuales o a realizar ciertas conductas sexuales que no desearan, un gran número de mujeres contestaban afirmativamente. Es decir, en un primer momento no consideraban que hubieran sido forzadas, sin embargo, sí que reconocían que habían sido obligadas. Lo mismo ocurría al explorar el método coercitivo empleado, con frecuencia primero afirmaban que sus maridos no les obligaban, pero si se profundizaba un poco más, referían que cuando ellas se negaban ellos respondían con violencia para coaccionarlas y conseguir su objetivo. Estas diferencias en las respuestas de las mujeres según la formulación de las preguntas, fue lo que llevó a considerar la necesidad de conocer los motivos de por qué no se profundizaba ante una información tan relevante.

Entre los posibles motivos algunos son culturales pero otros son profesionales. A nivel cultural sabemos que todavía en nuestra sociedad actual sigue imperando la idea del débito conyugal. De esta forma, algunas mujeres no son conscientes de que están siendo forzadas a practicar sexo, porque en último término consideran que es una obligación con su pareja (esté o no casada). Esta idea junto a los estereotipos femeninos, que entre otros muchos subrayan la pasividad y aceptación de las mujeres, conllevan a que sean las propias mujeres las que no reconozcan la propia situación de violencia sexual que estén viviendo con sus parejas. Hay que considerar que algunas de ellas también están siendo víctimas de otras acciones de violencia por parte de su pareja, lo que les hace entender que la violencia sexual es una forma más dentro de su relación. Es por todo ello que los profesionales tenemos que explorar activamente este tipo de violencia.

A nivel profesional se puede observar que las y los profesionales que trabajan con mujeres que han vivido violencia por sus parejas (personal sanitario, profesionales del ámbito

'Estos servicios de atención psicológica están enmarcados dentro de unos recursos públicos más completos de la Comunidad Valenciana. Uno de ellos es autonómico y atiende exclusivamente a mujeres que han sido víctimas de violencia de género. El otro servicio es municipal, atiende y asesora a cualquier mujer que esté empadronada en la localidad, a excepción de la atención psicológica, cuyo uso es exclusivo de las mujeres que han sufrido o sufren alguna forma la violencia de género. Ambos servicios son gratuitos, públicos, confidenciales y prestan asesoramiento y apoyo social, jurídico y psicológico. 


\section{dossier}

social-psicológico, cuerpos de seguridad, etc.), no suelen tener una formación especializada en sexología (Blanco, Ruiz-Jarabo, García y Martín-García, 2004; Menéndez, Pérez y Lorente, 2013), posiblemente entre otras cosas porque la oferta formativa en este campo es muy escasa (Borrás y Pérez, 2005; López, 2010). La falta de formación explicaría las reticencias a explorar en profundidad las relaciones sexuales de las mujeres con las que intervienen (Santana, Flores y Aguilar, 2004). Algunos autores como López (2014) van más allá y consideran que es inaceptable que, estando en el siglo XXI, las y los profesionales no exploren la violencia sexual por razones sexofóbicas, ancladas en una consideración de la sexualidad muy negativa y en prácticas educativas represivas. Por otro lado, se ha comprobado que las herramientas más utilizadas para la evaluación de la violencia en la pareja no son válidas para explorar la violencia sexual (Bagwell-Gray, Messing y BaldwinWhite, 2015). Al observar diferentes protocolos de entrevista publicados en español para la detección o valoración de la violencia sobre las mujeres en la relación de pareja, se puede comprobar que no exploran la violencia sexual en la relación. Tal es el caso, por ejemplo, de los protocolos publicados en Calvete, Corral y Estévez (2005), Labrador y Rincón (2002) o Martín, Ros, Garau, Mañes, Ponce y Mahiques (2006). Sólo algunos se limitan a hacer una pregunta genérica sobre agresiones sexuales, pero sin profundizar en las circunstancias específicas, como la frecuencia, duración, conductas realizadas, medio coactivo empleado o la respuesta de las mujeres. Este sería el caso de protocolos como los de Campbell (1995), Delgado, Aguar, Castellano y del Castillo (2006) o Echeburúa, Amor, Loinaz y Corral (2010). La única pregunta que se hace relacionada con el tema es si ha sufrido una agresión sexual por su pareja, dándose como opción una respuesta dicotómica, sí o no. En el caso de que no se explore nada más, podemos perder información muy importante sobre la situación real de este tipo de violencia y sobre la gravedad de la misma.

Esta ausencia llama especialmente la atención dado que la existencia de relaciones sexuales forzadas en la pareja es considerada un factor asociado de riesgo de homicidio y, por tanto, se puede considerar como un indicador de peligrosidad (Campbell, 1995; Echeburúa, Fernández-Montalvo y Corral, 2009; Labrador, Rincón, Luis de y Fernández-Velasco, 2008).

Otras herramientas exploran los abusos y/o las agresiones sexuales en mujeres, pero no recogen los casos en los que el agresor es la propia pareja, como ocurre en el modelo orientativo de entrevista dirigida a la mujer (Mestre, Tur y Samper, 2008), y por lo tanto, no se consideran aspectos como la posible habitualidad del abuso. La ausencia de exploración en las herramientas observadas, pone de manifiesto que, pese a que en los últimos años han proliferado las investigaciones sobre el maltrato físico y psicológico en la relación de pareja (Porrúa, Rodríguez-Carballeira, Almendros y Escartín, 2010), la violencia sexual es todavía una de las menos estudiada. Todo ello da pleno sentido a nuestro trabajo.

El marco teórico en el que podemos encuadrar la herramienta EVS que presentamos considera, en primer lugar, que la violencia sobre las mujeres en general y su relación de pareja en particular, deben considerarse un grave problema social, cuya visibilización y toma de conciencia han estado muy ligadas no sólo al discurso y avance de los movimientos feministas, sino también a la conquista de la libertad y de la democracia (Ferrer y Bosh, 2007). En segundo lugar, considera la salud sexual y reproductiva como un derecho humano, tal

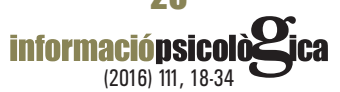




\section{dossier}

y como es definida por la Organización Mundial de la Salud (OMS, 2006) considerándola como "un estado de bienestar físico, emocional, mental y social relacionado con la sexualidad. No es solamente la ausencia de enfermedad, disfunción o discapacidad. Para que la salud sexual se logre y se mantenga, los derechos sexuales de todas las personas deben ser respetados, protegidos y ejercidos con plenitud". Por ello, la OMS asegura que se requiere un "enfoque positivo y respetuoso de la sexualidad y las relaciones sexuales, así como la posibilidad de tener relaciones sexuales placenteras y seguras, libres de coerción, discriminación y violencia". 'La sexualidad es un aspecto central de la calidad de vida del ser humano (Blümel, Binfa, Cataldo, Carrasco, Izaguirre y Sarrá, 2004) y la sexualidad femenina, una gran desconocida.

Sin embargo, la sexualidad es una diana contra la que se ejerce la violencia sobre las mujeres, en diferentes ámbitos y por diferentes agentes. En este sentido, el Informe Mundial sobre la Violencia y la Salud determina que: "la violencia sexual comprende una gran diversidad de actos, como las relaciones sexuales bajo coacción en el matrimonio y en las citas, las violaciones por parte de extraños, las violaciones sistemáticas durante los conflictos armados, el acoso sexual (incluida la petición de favores sexuales a cambio de trabajo o calificaciones escolares), los abusos sexuales de menores, los matrimonios precoces y los actos violentos contra la integridad sexual de las mujeres, como la mutilación genital y las inspecciones obligatorias de la virginidad“ (Organización Panamericana de la Salud Sexual, 2002, p.21).

Entre las diferentes formas de violencia sexual, podemos diferenciar entre Agresión Sexual y Abuso Sexual. Por un lado, se entiende por Agresión Sexual aquellos actos que atentan contra la libertad sexual de las personas, implicando violencia e intimidación y no consentimiento de la víctima, y por supuesto incluye las agresiones sexuales aun cuando el atacante sea la pareja sentimental o el marido (García, Canet, Gascó, Ribelles, Abellán y Prats, 2001). Por otro lado, el Código Penal tipifica como Abuso Sexual el que, sin violencia o intimidación y sin que medie consentimiento, realizare actos que atenten contra la libertad o indemnidad sexual de otra persona, y se entiende que no hay consentimiento, no sólo cuando la víctima se halle privada de sentido o de cuyo trastorno mental se abusare, también los que se cometan anulando la voluntad de la víctima mediante el uso de fármacos, drogas o cualquier otra sustancia natural o química idónea a tal efecto, sino cuando el consentimiento se obtenga prevaliéndose el responsable de una situación de superioridad manifiesta que coarte la libertad de la víctima (Ley Orgánica 10/1995, de 23 de noviembre, del Código Penal Art. 181). Estas dos formas de violencia se pueden dar tanto dentro como fuera de la relación de pareja, si bien el abuso sexual es la que más se da en las situaciones de violencia en la pareja, en la que un hombre tiene una posición de superioridad respecto de una mujer y ejerce el control sobre su sexualidad. Además, se ha observado que a menudo, los agresores exigen tener relaciones sexuales después de un episodio de violencia física, lo que supone una nueva agresión para las mujeres dado que, en la situación psíquica y emocional en que se encuentran, no son libres para prestar su consentimiento (Martín, Fernández y Chato, 2006). Sin embargo, de forma errónea, cuando oímos hablar de agresión sexual se tiende a pensar exclusivamente en una violación que se ejerce junto a una agresión física brutal, 


\section{dossier}

puntual y/o llevada a cabo por un desconocido. Cuando se habla de abuso sexual, se entiende erróneamente sólo el que sufre un/a menor de edad o persona discapacitada.

Por ello, de entre los diversos conceptos que se manejan en la literatura científica actual para definir la violencia sexual sobre las mujeres en la relación de pareja, en el presente trabajo asumimos la definición de maltrato sexual que proponen Labrador et al. (2008, p. 26); "cualquier intimidad sexual forzada por parte de la pareja, ya sea con amenazas, intimidación, coacción, o por llevarse a cabo en estado de inconsciencia o indefensión de la mujer, incluye todo tipo de conducta de carácter sexual, no sólo la penetración vaginal o anal".

En cuanto a la prevalencia de la violencia sexual en la relación de pareja en población normal existen pocas investigaciones y las tasas encontradas oscilan entre el 2,4\% y el 59\% de la población (Martínez-Sanz y Mañas, 2014). Esta variabilidad en las cifras es el resultado, en gran medida, de la falta de acuerdo en la terminología utilizada y en la definición de violencia sexual (Bagwell-Gray, et al., 2015).

En lo que sí hay acuerdo en la literatura es sobre las consecuencias sobre las mujeres de la violencia sexual en la relación de pareja. Se pone de manifiesto que el carácter humillante y vejatorio de la violencia sexual aumenta la gravedad del trastorno de estrés postraumático (Amor, Echeburúa, Corral, Zubizarreta y Sarasúa, 2002; Benice, Resick, Mechanic y Astin, 2003; Dutton, Kalman, Goodman, Weinfun y Vankos, 2005; Merlin y Mohr, 2000). Se ha encontrado una asociación positiva con la existencia de mayor depresión, mayor ideación suicida, mayor número de intentos autolíticos y mayor consumo de psicofármacos (Fischbach y Herbert, 1997; Ramos-Lira, et al., 2001). Las consecuencias físicas de la violencia sexual están relacionadas con problemas ginecológicos (Campbell, 2002). Además, la violencia sexual repercute en otros aspectos de la salud sexual y reproductiva (Apt y Hurlbert, 1993; DeMaris, 1997; Dutton, 1992; Ramírez y Patiño, 1997; Santos-Iglesias y Sierra, 2012; entre otros).

En definitiva, cuando se sostiene una relación de abuso y desequilibrio de poder donde reina el miedo y una mujer tiene coartada su libertad para tomar decisiones, probablemente no tendrá potestad para decidir sobre su sexualidad y aumentará la probabilidad de sufrir violencia sexual por su pareja. La violencia sexual, ya sea por su extorsión, por su coexistencia, o por los efectos que puede tener sobre el estado psíquico y emocional de la víctima, como cualquier tipo de violencia, no se da aislado. Se repite a lo largo de la relación de pareja y afecta de forma integral a toda la persona. Por ello, consideramos muy importante explorar la violencia sexual, porque así se podrá conocer mejor la realidad, aumentar la empatía y mejorar la intervención. Las y los profesionales debemos tener un papel activo en la restructuración de los estereotipos que constituyen un sistema encubierto y silenciador que coloca a las mujeres en una situación de vulnerabilidad por el mero hecho de ser mujer (García, 2010). La EVS que en este trabajo se presenta pretende contribuir a constatar y explorar con mayor sistematicidad y rigor la violencia sexual en la pareja.

El objetivo del estudio era crear una herramienta que permitiera conocer la posible violencia sexual que haya sufrido o esté sufriendo una mujer por parte de su pareja. 


\section{dossier}

\section{Método}

\section{Instrumento}

Para evaluar la violencia en la pareja, se consideró que la mejor fórmula era la entrevista semiestructurada (Porrúa, et al., 2010). La entrevista es un instrumento clínico, complementario a otras técnicas de obtención de información, que permite obtener datos sobre la vida de una persona. En concreto, la entrevista semiestructura permite guiar la obtención de información sobre cuestiones específicas que se pretenden conocer, combinando preguntas abiertas, que permiten flexibilidad y matices en las respuestas, con otras más cerradas que no obstante dejan margen para que el entrevistado pueda salirse del "guión" (Bleger, 1964/1985).

La entrevista que proponemos además es individual (porque se realiza a una persona), focalizada (porque se centra en un aspecto o tema concreto) y profunda (porque a medida que se avanza en la entrevista se va evidenciando aspectos más íntimos). Al mismo tiempo, la entrevista puede ser terapéutica en sí misma, debiendo cuidar aspectos tan importantes como la comunicación no verbal y la creación de un espacio seguro y confidencial que facilite la expresión emocional (Russel, 2006; Massot, Dorio y Sabariego, 2004).

Teniendo en cuenta estos aspectos, la EVS se concibió como un instrumento que facilitara a las y los profesionales relacionados con el tema la exploración de este tipo de violencia. Esta entrevista va dirigida principalmente a las mujeres que refieren haber vivido algún tipo de violencia por parte de sus parejas y acuden a un servicio especializado demandando asesoramiento y apoyo. Además, puede ser utilizada en cualquier otro contexto en el que sea de interés clínico, conocer si las mujeres entrevistadas presentan o no abuso sexual por parte de su pareja.

Se opta por una entrevista breve porque se considera que es un instrumento complementario a otras técnicas de obtención de información. Es decir, previamente se habrá preguntado a la mujer sobre sus datos personales, familiares, su historia de vida, así como se habrá explorado el posible maltrato físico y/ psicológico, u otras circunstancias estresantes en las que se encuentre. Por lo tanto, forma parte de un contexto amplio de evaluación de las mujeres que acuden a estos servicios.

\section{Procedimiento seguido en la elaboración de la EVS}

Para la construcción de los ítems que constituyen la base estructural de esta herramienta procedimos a realizar una búsqueda de trabajos y material relevante a partir de una revisión de la bibliografía relacionada con el tema, (ver Apéndice 1). La búsqueda se realizó en las bases de datos online especializadas en Psicología o en materias afines: PsycINFO, PsicoDoc, SCOPUS, ProQuest Psychology, PsycARTICLES, PudMed y Dialnet. El criterio de selección se centró en artículos y protocolos que incluyeran algún aspecto relacionado con la evaluación del abuso sexual y especialmente en el ámbito de la violencia contra la pareja. Se descartaron las publicaciones meramente jurídicas o médicas. También se realizó una búsqueda manual y se examinaron referencias secundarias.

El objetivo de la entrevista era que, de una forma sencilla y breve, se pudiera tener conocimiento de si las mujeres evaluadas estaban o no sufriendo una situación de violencia sexual

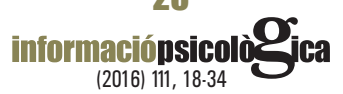




\section{dossier}

por parte de sus parejas, así como conocer las características principales de esta violencia. Teniendo en cuenta este objetivo, de los trabajos encontrados se seleccionaron aquellos que pudieran aportar información o ítems relacionados con los criterios que se habían establecido y que se detallan a continuación:

Identificar algunas cuestiones sobre la salud sexual y reproductiva de las mujeres entrevistadas. Para ello se tuvieron en cuenta alguna de las cuestiones que plantea Souza y sus colaboradores (1987): si utilizan algún método anticonceptivo y en caso afirmativo especificar el método utilizado; si alguna vez se han quedado embarazadas sin planificarlo previamente, con el objetivo de conocer si las parejas han impedido o dificultado la prevención de embarazos no deseados; si han sufrido alguna interrupción voluntaria del embarazo, para saber si se han visto obligadas a interrumpir el embarazo o ha sido una decisión propia; si han llevado un control ginecológico regular; si han tenido alguna enfermedad de transmisión sexual (ETS) y si el contagio ha sido causado por las parejas.

Explorar si han sufrido en el pasado alguna agresión sexual o abuso sexual por otra/s persona/s. Se pretende conocer de forma exploratoria si existen antecedentes de violencia sexual y las circunstancias de los mismos (Martínez-Sanz, Mañas e Iniesta, 2014).

Conocer si en alguna ocasión las mujeres han sido humilladas por haber llevado la iniciativa sexual. Habitualmente a las mujeres se les ha adjudicado un papel pasivo en el sexo. No ha estado bien visto que accedieran al placer y las que lo han buscado, han sido objeto de todo tipo de críticas y juicios peyorativos, un juicio muy diferente del que por idénticos motivos han merecido los hombres (Asensio y Tomas, 2000). En algunas ocasiones en las entrevistas, las mujeres informan que cuando llevan la iniciativa para iniciar una relación sexual, su pareja las ha castigado con menosprecios y/o insultos.

Conocer si las mujeres se han visto obligadas a mantener relaciones sexuales con sus parejas o a realizar conductas sexuales que no desearan. Tal y como se ha indicado, la violencia sexual en la relación de pareja no suele ser percibida, y por ello no es explorada. La violencia sexual sobre las mujeres, incluye todo acto sexual o la tentativa de consumarlo, los comentarios o insinuaciones sexuales no deseados o las acciones destinadas a la utilización de cualquier otro modo se la sexualidad de una mujer mediante coacción por parte de otra persona, independientemente de la relación de ésta con la víctima (Jewkes, Sen y GarcíaMoreno, 2002).

Concretar el tipo de conductas sexuales a las que se han visto obligadas a realizar. La violencia sexual comprende cualquier actividad sexual no consentida y por ello interesa conocer el tipo de conducta sexual forzada. Para establecer las diferentes acciones de abuso se han seguido las propuestas realizadas por varios autores y trabajos (Dutton, 1992; Centro Mujer 24 Horas, 2003; Mestre, et al., 2008; Protocolo marco de actuación profesional, 2008). La lista incluye: tocamientos sexuales, penetración vaginal o anal, sexo oral, la exposición de genitales y/o cuerpo desnudo, observación de revistas, películas pornográficas o a otras personas desnudas o realizando actividad sexual, usar objetos o ropas con fines sexuales, mantener relaciones sexuales con otras personas incluyendo a menores o incluso con animales, y filmación o realización de fotografías de la actividad sexual. 


\section{dossier}

Conocer el medio coercitivo empleado por sus parejas para obligarlas a mantener relaciones sexuales. Conocer los medios utilizados para la coacción puede ayudar a reconocer cuáles son las circunstancias de este tipo de violencia, así como la gravedad de la misma. Finkelhor e Yllö (1983) proponen cuatro tipos de métodos de coacción que utilizan los hombres para obligar a las mujeres sexualmente: coerción social, coerción interpersonal, amenaza con la fuerza física y coacción física. Dutton (1992) señala también cuatro tipos: el uso de fuerza física, amenazas con causar daño físico a la mujer o a otra persona, amenazas con consecuencias negativas y presión social (expectativas sexuales propias o de otros).

Indagar sobre la respuesta que las mujeres han dado ante estas situaciones. Las mujeres responden de diversas formas ante la coacción ejercida por sus parejas para mantener relaciones sexuales. Estas respuestas son importantes conocerlas para poder realizar una valoración del nivel de riesgo de que la violencia sexual se mantenga. Estas respuestas van desde la negación explícita, la respuesta pasiva, fingir placer, llorar y/o participar de forma activa (Martínez-Sanz, et al., 2013).

Explorar la frecuencia con la que ocurren estos hechos y durante cuánto tiempo se ha dado esta situación. Generalmente no son sucesos aislados sino que se repiten a lo largo de meses incluso años, provocando en la víctima no sólo daños físicos, sino sobre todo afectando gravemente a su estabilidad psicológica (Amor, Echeburúa, Corral y Zubizarreta, 2001; Cáceres, 2002; Cáceres y Cáceres, 2006; Sarasúa, Zubizarreta, Echeburúa y Corral, 2007). Por ello, interesa conocer la frecuencia y la duración de la violencia sexual sobre las mujeres en la relación de pareja.

Considerar otra información relevante que no se haya explorado pero que sea significativa y que esté relacionada con la violencia sexual. Es importante dejar un espacio para que las y los profesionales puedan indicar aquellos aspectos que las mujeres han verbalizado en el transcurso de la entrevista y que no se hayan recogido en las cuestiones anteriores.

La entrevista resultante se presenta en el Apéndice 2 del artículo.

\section{Conclusiones}

La violencia sexual que se ejerce sobre las mujeres en la relación de pareja es vivida por un gran número de mujeres de nuestro país y de todo el mundo (Mahoney, et al., 2007). Esta violencia conlleva graves consecuencias físicas, psicológicas y sexuales-reproductivas para las mujeres que la sufren (Villavicencio y Sebastian, 2001). Sin embargo, no suele ser investigada (Porrúa, et al., 2010), ni tampoco explorada por las y los profesionales que trabajan con mujeres que han vivido violencia en la pareja. Además, las propias mujeres que la sufren, no suelen percibirla como tal, por lo que se mantiene oculta de modo que esta invisibilidad favorece a que se perpetúe.

Por ello, la Entrevista Semiestructurada para la Exploración de la Violencia Sexual sobre las Mujeres en la Relación de Pareja (EVS), es una herramienta que ha sido diseñada para obtener información sobre las características de la violencia sexual en la relación de pareja y las circunstancias en las que ocurre. Está pensada para que sea utilizada como un "guión" de apoyo orientativo para profesionales, para obtener información preguntando directamen- 


\section{dossier}

te, aunque siempre es preferible que, en la medida de lo posible, sean las mujeres quienes cuenten sus historias de violencia de la forma más abierta posible, es decir, de manera espontánea, y en un contexto de confianza y empatía en el que se propicie la expresión emocional.

La EVS explora cuestiones sobre la salud sexual y reproductiva, los antecedentes de violencia sexual en el pasado, si las mujeres han sido humilladas por haber llevado la iniciativa sexual, si se han visto obligadas a realizar conductas sexuales que no desearan, qué conductas específicas se han visto obligadas a realizar, qué métodos de coacción han utilizado sus parejas, cómo han respondido ellas ante esta violencia, la frecuencia y la duración de la misma, así como contempla un apartado para recoger otro tipo de información que se consideren relevantes relacionada con la violencia sexual y no conste de manera explícita en la EVS.

Por tanto, la EVS pretende ser una herramienta que facilite, por un lado, la exploración profesional y, por otro lado, el relato de las mujeres que han vivido violencia sexual en la relación de pareja. De esta manera, esperamos contribuir en una mayor detección y conocimiento de este tipo de violencia, y por tanto, dar un primer paso hacia la erradicación, porque no se puede luchar contra lo que no se conoce.

\section{Referencias}

Amor, P., Echeburúa, E., Corral de, P., Sarasúa, B., y Zubizarreta, I. (2001). Maltrato físico y maltrato psicológico en mujeres víctimas de violencia en el hogar: un estudio comparativo. Revista de Psicopatología y Psicología Clínica, 6, 167-178. doi: http://dx.doi.org/10.5944/rppc.vol.6.num.3.2001.3913

Amor, P., Echeburúa, E., Corral de, P., Zubizarreta, I., y Sarasua, B. (2002). Repercusiones psicopatológicas de la violencia doméstica en la mujer en función de las circunstancias del maltrato. Revista Internacional de Psicología Clínica y Salud/ International Journal of Clinical and Health Psychology, 2, 227-246.

Apt, C., y Hurlbert, D. F. (1993). The sexuality of women in physically abusive marriages: A comparative study. Journal of Family Violence, 8, 57-69. doi: 10.1007/BF00986993

Asensio. F., y Tomás, T. (2000). Tratado médico familiar de la sexualidad en la mujer. Anatomía, fisiología, patología. Barcelona: Guías Atrium de medicina y salud.

Bagwell-Gray, M. E., Messing, J. T., y Baldwin-White, A. (2015). Intimate partner sexual violence: A review of terms, definitions, and prevalence. Trauma Violence \& Abuse, 16 (3): 316-335. doi: 10.1177/1524838014557290

Benice, J. A., Resick, P.A., Mechanic, M., y Astin, M. (2003). The relative effects of intimate partner physical and sexual violence on post-traumatic stress disorder syntomatology. Violence and Victims, 18, 87-94.

Blanco, P., Ruiz-Jarabo, C., García de Vinuesa, L., y Martín-García, M. (2004). La violencia de pareja y la salud de las mujeres. Gaceta Sanitaria, 18 (Supl.1), 182-188.

Bleger, J. (1985). La entrevista psicológica. Su empleo en el diagnóstico y en investigación. Buenos Aires: Universidad de Buenos Aires.

Blümel, J. E., Binfa, L., Cataldo, P., Carrasco, A., Izaguirre, H., y Sarrá, S. (2004). Índice de Función Sexual Femenina: Un test para evaluar la sexualidad de la mujer. Revista Chilena de Obstetricia y Ginecología, 69 (2), 118-125.

Borrás, J. J., y Pérez, M. (2005). Formación de especialistas en sexología en España y Latinoamérica. Sexología Integral, 2 (3), 144-152. 


\section{dossier}

Cáceres, J. (2002). Análisis cuantitativo y cualitativo de la violencia doméstica en la pareja. Cuadernos de Medicina Psicosomática y Psiquiatría de Enlace, 60-61, 57-67.

Cáceres, J. (2004). Violencia física, psicológica y sexual en el ámbito de la pareja: Papel del contexto. Clínica y Salud, 15 (1), 33-54.

Cáceres, A., y Cáceres, J. (2006). Violencia en las relaciones íntimas en dos etapas evolutivas. Internacional Journal of Clinical and health Psychology, 6 (2), 271-284.

Calvete, E., Corral, S., y Estévez, A. (2005). Desarrollo de un inventario para evaluar el abuso psicológico en las relaciones de pareja. Clínica y Salud, 16 (3), 203-221.

Campbell, J. C. (1995). Danger assessment toll (DA). En E. Echeburúa, J. Fernández-Montalvo, y P. de Corral de (Eds.). (2009). Predicción del riesgo de homicidio y de violencia grave en la relación de pareja. Instrumentos de evaluación del riesgo y adopción de medidas de protección. Centro Reina Sofía. Generalitat Valenciana.

Campbell, J. C. (2002). Health consequences of intimate partner violence. The Lancet, 359, 1331-1336. doi: http:// dx.doi.org/10.1016/S0140-6736(02)08336-8

Carvalheira, A. A. (2007). Intervención terapéutica en las disfunciones sexuales femeninas: perspectiva psicosexológica. Revista Internacional Andrológica, 5 (1), 88-91. doi:10.1016/S1698-031X(07)74038-4

Delgado, A., Aguar, M., Castellano, M., y Castillo del, J. D. L. (2006). Validación de una escala para la medición de los malos tratos a mujeres. Atención Primaria, 38 (2), 82-89. doi: 10.1157/13090429

DeMaris, A. (1997). Elevated sexual activity in violent marriages: hypersexaulity or sexual extorsion? The Journal of Sex Research. 34 (4), 361- 373. doi: 10.1080/00224499709551904

Dutton, M. A. (1992). Empowering and healding the battered woman. New York: Springer Publishing Company.

Dutton, M. A., Kalman, S., Goodman, L. A., Weinfun, K., y Vankos, N. (2005). Patterns of intimate partner violence: Correlates and outcomes. Violence and Victims, 20, 483- 497. doi: http://dx.doi.org/10.1891/08866708.2005.20.5.483

Echeburúa, E., Amor, P. J., Loinaz, I., y Corral de, P. (2010). Escala de predicción del riesgo de violencia grave contra la pareja. Revisada. (EPV-R). Psicothema, 22 (4), 1054-1060.

Echeburúa, E., Corral, P., Sarasua, B., Zubizarreta, I. y Sauca. (1994). Entrevista semiestructurada para víctimas de maltrato doméstico. En E. Echeburúa, y P. Corral (Eds.). (2006). Manual de violencia familiar. Madrid: Siglo XXI.

Echeburúa, E., Fernández-Montalvo, J., y Corral, P. (2009). Predicción de riesgo de homicidio y de violencia grave en la relación de pareja. Instrumentos de evaluación del riesgo y adopción de medidas de protección. Serie Documentos 15. Valencia: Centro Reina Sofía. Generalitat Valenciana.

Equipos profesionales de los Centro Mujer 24 Horas de Alicante, Castellón y Valencia. (2003). Intervenciones con mujeres víctimas de violencia de género, 2000. Valencia: Conselleria de Benestar Social.

Ferrer, A., y Bosh, E. (2007). El papel del movimiento feminista en la consideración social de la violencia contra las mujeres: el caso de España. Revista de Estudios Feministas, 10 (Dossier España).

Finkelhor, D., y Yllö, K. (1983). Rape in marriage: a sociological view. En D., Finlelhor, R. J., Gelles, y M. A. Strauss (Eds.). The dark side of families: current family violence research. Newbury: Sage.

Fischbach. R. L., y Herbert, B. (1997). Domestic violence and mental Health: correlates and conundrums within and across cultures. Social Science and Medicine, 45 (8), 1161-1176. doi: 10.1016/S0277-9536(97)00022-1

García, M. (2010). Aspectos psicológicos sobre la violencia de género. En N. Menchal Escalona (Ed.). (2010). Manual de lucha contra la violencia de género. Navarra: Editorial Aranzadi.

García, E., Canet, E., Gascó, E., Ribelles, A., Abellán, C., y Prats, L. (2001). Manual sobre la violencia contra las mujeres. Guía de información para la mujer, en situación de Abuso. Valencia: Conselleria de Benestar Social.

Jewkes, R., Sen, P., y Garcia-Moreno, C. (2002). Sexual violence. En E. G. Krug, L. L. Dahlberg, J. A. Mercy, A. B. Zwi, y R. Lozano (Eds.). World report on violence and health (pp. 149-181). Ginebra: Organización Mundial de la Salud. 


\section{dossier}

Entrevista semiestructurada para la exploración de la violencia sexual sobre las mujeres en la relación de la pareja (EVS).

Labrador, F. J., Fernández-Velasco, M. R., y Rincón, P. (2010). Características psicopatológicas de mujeres víctimas de violencia de pareja. Psicothema, 22 (1), 99-105.

Labrador, F. J., y Rincón, P. P. (2002). Pauta de entrevista para víctimas de violencia doméstica. En F.J. Labrador, P.P. Rincón, P. de Luis, y R. Fernández-Velasco (Eds.). (2008). Mujeres víctimas de la violencia doméstica. Programa de actuación (pp. 190-191). Madrid: Psicología- Pirámide.

Labrador F. J., Rincón, P. P., Luis de, P., y Fernández-Velasco, R. (2008). Mujeres víctimas de la violencia doméstica. Programa de actuación. Madrid: Psicología- Pirámide.

Lameiras, M., Carrera, M V., y Rodríguez, Y. (2013). Sexualidad y salud. El estudio de la sexualidad humana desde una perspectiva de género. Universidad de Vigo.

Ley Orgánica 10/1995, de 23 de noviembre, del Código Penal. Art. 181. En BOE núm. 281, de 24 de Noviembre de 1995 (pp. 33987-34058).

López, F. (2010). Estudios sobre sexualidad en España: presente y futuro. Información psicológica, 100, 84-90.

López, F. (2014). Los abusos sexuales a menores y otras formas de maltrato sexual. Madrid: Editorial Síntesis. Manual de Prevención y Recursos ante la violencia de Género. (2000). Área Mujer. Diputación de Alicante.

Mahoney, P., y Williams, L. M. (2007). Sexual assault in marriage: Prevalence, consequences, and treatment of wife rape. Partner Violence: A 20-year Literature Review and Synthesis, 1-43.

Mañez, P. Ponce, M., Mahiques, E., Martín, M., Ros, M., y Garau, J. (2006). Manual de Buenas Prácticas. Gestión de Centros de Acogida a Mujeres de la Comunidad Valenciana. Generalitat Valenciana.

Martínez-Sanz, A., y Mañas, C. (2015). La violencia sexual sobre la mujer dentro de la relación de pareja. En M.M. Lafaurie y M.A. Sánchez (Comp.). Salud sexual y reproductiva: nuevas tendencias. Bogotá: Universidad El Bosque.

Martínez-Sanz, A., Mañas, C., y Iniesta, A. (2014). Antecedentes de violencia en la infancia y sintomatología en mujeres que han vivido violencia por sus parejas. INFAD Revista de Psicología, 1 (3): 431-438.

Martínez-Sanz, A., Mañas, C., y Montesinos, N. (2013). Maltrato sexual sobre las mujeres en sus relaciones de pareja: un estudio de caso. Asparkía, 24, 88-103.

Massot, I., Dorio, I., y Sabariego, M. (2004). Estrategias de recogida y análisis de la información. En R. Bisquerra (ed.), Metodología de la investigación educativa (pp. 329-366). Madrid: La Muralla.

Matud, M. P., Padilla, V., y Gutiérrez, A. B. (2009). Mujeres maltratadas por su pareja. Guía de tratamiento psicológico. ( $2^{\mathrm{a}}$ ed.). Madrid: Minerva Ediciones.

Menéndez, S., Pérez, J., y Lorence, B. (2013). La violencia de pareja contra la mujer en España: Cuantificación y caracterización del problema, las víctimas, los agresores y el contexto social y profesional. Psychosocial Intervention, 22 (1), 41-53. doi:10.5093/in2013a6

Merlin, P., y Mohr, P.B. (2000). Incidence and correlates of postraumatic stress disorder in Australian victims of domestic violence. Journal of Family Violence. 15, 411-422.

Mestre, M. V., Tur, A. M., y Samper, P. (2008). Impacto psicosocial de la violencia de género en las mujeres y sus hijos e hijas. Un estudio empírico en la Comunidad Valenciana. Fundación de la Comunidad Valenciana frente a la discriminación y malos tratos Tolerancia Cero (Conselleria de Benestar Social) y la Universitat de Valencia.

Organización Mundial de la Salud. (2005). Estudio multipaís de la OMS sobre la salud y la violencia doméstica: primeros resultados sobre prevalencia, eventos relativos a la salud y respuestas de las mujeres a dicha violencia: Resumen del informe. Ginebra: Autor.

Organización Mundial de la Salud (2006). Defining sexual health: report of a technical consultation on sexual health, 28-31 January 2002, Geneva.

Organización Panamericana de la Salud. (2002). Informe mundial sobre la violencia y la salud. Resumen. Washington, D. C.: Oficina Regional para las Américas de la Organización Mundial de la Salud.

Porrúa, C., Rodríguez-Carballeira, A., Almendros, C., y Escartín, J. (2010). Análisis de las estrategias de abuso psicológico en la violencia en la pareja. Información Psicológica, 99, 53-63. 


\section{dossier}

Protocolo marco de actuación profesional para casos de violencia de género en Castilla y León. (2008). Junta de Castilla y León.

Ramírez, J. C., y Patiño, M. C. (1997). Algunos aspectos sobre la magnitud y trascendencia de la violencia doméstica contra la mujer: un estudio piloto. Salud Mental, 20 (2), 5-16.

Ramos-Lira, L., Saltijeral-Méndez, M. T., Romero-Mendoza, M. Caballero-Gutiérrez, M. A., y Martínez-Vélez, N. A. (2001). Violencia sexual y problemas asociados en una muestra de usuarias de un centro de salud. Salud Pública de México, 43 (3), 182-191.

Russel, B. H. (2006). Research methods in antthropology. Oxford: Rowman y Litlefield Pub.

Santana, F., y Calero, J. L. (2004). Selección del sexo: algunas reflexiones desde la ética. Revista Cubana de Salud Pública, 30 (3). Recuperado en 2 de junio de 2016, de http://scielo.sld.cu/scielo.php?script=sci_ arttext\&pid=S0864-34662004000300012\&lng=es\&tlng=es

Sarasua, B., Zubizarreta, I., Echeburúa, E., y Corral, P. (2007). Perfil psicopatológico diferencial de las víctimas de violencia de pareja en función de la edad. Psicothema, 19 (3), 459-466.

Santos-Iglesias, P., y Sierra, J.C. (2012). Sexual Victimization among Spanish College Women and Risk Factors for sexual Revictimization. Journal of Interpersonal Violence, 27 (17), 3468-3485. doi: 10.1177/0886260512445383

Sierra, J. C., Santos, P., Gutiérrez-Quintanilla, J. R., Gómez, P., y Maeso, M. D. (2008). Un estudio psicométrico del Hurlbert Index of Sexual Assertiveness en Mujeres Hispanas. Terapia Psicológica, 26 (1), 117-123. doi: http:// dx.doi.org/10.4067/S0718-48082008000100010

Souza, M., Cárdenas, J., Montero, M. C., y Mendoza, L. (1987). Historia clínica codificada para la sexualidad femenina. Ginecología y Obstetricia de México, 55 (octubre), 277-287

Valdez-Santiago, R., y Sanín-Aguirre, L. H. (1996). La violencia doméstica durante el embarazo y su relación con el peso al nacer. Salud Pública de México, 38 (5), 352-362.

VanZile-Tamsen, C. (2007). The reciprocal relationship between sexual victimization and sexual assertiveness. Violence against Women, 13 (3), 298-313. doi: 10.1177/1077801206297339

Villavicencio, P., y Sebastián J. (1999/2001). Violencia doméstica: Su impacto en la salud física y mental de las mujeres. Madrid: Instituto de la Mujer. Ministerio de trabajo y asuntos sociales. 


\section{dossier}

Apéndice 1: Material y publicaciones que recogen algún aspecto de la violencia sexual en la pareja.

1. Herramientas que exploraran el maltrato físico, psicológico, emocional, económico y que incluyan también algunos items sobre la violencia sexual.

1.1.Índice de violencia "Me fuerza a hacer actos sexuales que no me gustan" en la pareja (Hudson "Me exige relaciones sexuales, aunque esté cansada" y Mcintosh, 1981). Adaptación J. Cáceres (2004).

1.2. Evaluación sobre la "Tipo de abuso experimentado: físico/psíquico/sexual" violencia contra la pa- "Actos cometidos en el abuso: ... agresiones sexuareja (Echeburúa et al., les..." 1994, 2006). “Mantiene relaciones sexuales actualmente?”

1.3. Evaluación de la his- “¿Ha sufrido abusos sexuales?” toria y tipo de maltrato (Matud et al., 2009).

1.4. Escala de violencia e “ ¿Le ha exigido tener relaciones sexuales con él? ¿ $\mathrm{Ha}$ índice de severidad para usado la fuerza física para tener relaciones sexuales con medir violencia de pa- usted? ¿Ha amenazado con irse con otras mujeres si no reja en mujeres mexica- accede a tener relaciones sexuales?"

nas (Valdez-Santiago et al. 2006).

1.5. Estudio sobre magni- Incluye cuestionario que recoge preguntas relacionadas tud y trascendencia de con la violencia sexual en la pareja: ¿Le ha exigido tela violencia doméstica ner relaciones sexuales sin importar si usted lo desea?, (Ramírez, et al., 1997). ¿La ha forzado, utilizando la fuerza física, a tener relaciones sexuales contra su voluntad?, ¿La ha obligado al sexo oral contra su voluntad?

2. Herramientas que exploran la agresión sexual (como violación puntual).

2.1. Modelo orientativo Se pregunta por antecedentes de abusos sexuales y de entrevista dirigida a cuestiones relacionadas con el informe médico. la mujer (Mestre et al., 2008).

2.2. Publicación de la Con- Se recogen datos estadísticos sobre las condiciones esselleria de Benestar So- pecíficas en las que se dan los abusos sexuales y las cial de la Generalitat. agresiones sexuales sufridas por las mujeres (Centro Mujer 24 Horas de Alicante, Castellón y Valencia).

3. Trabajos que conceptualizan la violencia sexual sobre las mujeres. 
3.1. Estudio multi-país de la OMS sobre la salud y la violencia doméstica (2005).
Define la violencia sexual sobre las mujeres y explica que tiene cabida en el ámbito de la pareja afectiva.

3.2. Blanco, et al. (2004). Destacan consecuencias de la violencia sobre la salud sexual y reproductiva de las mujeres como disminución de la líbido, anorgasmia, falta de autonomía sexual, embarazos no deseados, de alto riesgo y complicaciones, ETS, abortos en condiciones inseguras, infecciones urinarias de repetición y dolores pélvicos crónicos.

3.3. Finkelhor e Yllö (1983) Describen la situación de violencia sexual en la pareja y reconocen cuatro tipos de coerción utilizadas en estas situaciones: coerción social, interpersonal, amenazas con fuerza física y coacción física.
3.4. Valdez-Santiago et al., Describen otras situaciones de coacción y exigencias (2006) para conseguir relaciones sexuales en la pareja como son las amenazas con irse con otras mujeres y el uso de la fuerza física.

3.5.Lameiras, Carrera y Ro- Afirman que dentro de la violencia sexual sobre las mudríguez (2013) jeres se incluyen aquellas que ocurren en la relación de pareja y destacan la ausencia de reconocimiento de este problema por pate de la sociedad.

4. Protocolos y guías de actuación ante la violencia de género.

4.1. Protocolo marco de Cita la clasificación de los diferentes tipos de violenactuación profesional para casos de violencia cia que pueden sufrir las mujeres según el Consejo de de género en Castilla y León (2008) Europa. Especifica que la violencia sexual comprende cualquier actividad sexual no consentida (visionado o participación forzada en pornografía, relaciones sexuales obligadas, etc.).

4.2. Martín et al. (2006). Dentro de "la tipología de la violencia de género" incluye la "violencia sexual", indicando que es la participación forzada en cualquier tipo de actividad sexual. "Nadie, ni siquiera el cónyuge o compañero, tiene derecho a obligar a una mujer a tener relaciones sexuales si ella no quiere". 
4.3. Manual de prevención Afirma que "los abusos y las agresiones sexuales son de recursos ante la vio- actitudes y comportamientos de carácter sexual que se lencia de género (2000). realizan sin consentimiento de la víctima, con indepenExcmo. Diputación de dencia de que se emplee la manipulación emocional o Alicante. Área Mujer. la violencia física". Se considera que no son sucesos aislados sino que se repiten a lo largo de meses incluso años, provocando en la víctima daños físicos y psicológicos.

5. Historia clínica para la sexualidad femenina.

5.1. Souza et al. (1987) Describe la sexualidad de las mujeres y propone cuestiones interesantes que pueden servir para la exploración de la violencia sexual en la pareja.

Apéndice 2. Entrevista Semiestructurada para la Exploración de la Violencia Sexual sobre las Mujeres en la Relación de Pareja.

\begin{tabular}{|c|c|c|}
\hline \multirow{2}{*}{$\begin{array}{l}\text { ¿Utilizas algún tipo } \\
\text { método anticoncep- } \\
\text { tivo? }\end{array}$} & Sí & ¿Qué tipo? \\
\hline & No & ¿Por qué motivo? \\
\hline \multirow{2}{*}{$\begin{array}{l}\text { ¿Te has quedado em- } \\
\text { barazada sin planifi- } \\
\text { carlo previamente? }\end{array}$} & Sí & ¿Qué ocurrió? \\
\hline & No & \\
\hline \multirow{2}{*}{$\begin{array}{l}\text { ¿Has tenido alguna } \\
\text { interrupción volunta- } \\
\text { ria del embarazo? }\end{array}$} & Sí & $\begin{array}{l}\text { ¿Cuántas veces? ¿Qué ocurrió? ¿Tuviste el apoyo } \\
\text { de tu pareja? ¿Te obligó tu pareja a realizar la inte- } \\
\text { rrupción? }\end{array}$ \\
\hline & No & \\
\hline \multirow{2}{*}{$\begin{array}{l}\text { ¿Has tenido algún } \\
\text { aborto espontáneo? }\end{array}$} & Sí & ¿Cuántas veces? ¿Qué ocurrió? \\
\hline & No & \\
\hline \multirow{2}{*}{$\begin{array}{l}\text { ¿Te realizas revisio- } \\
\text { nes ginecológicas } \\
\text { regularmente? }\end{array}$} & Sí & ¿Con qué frecuencia? \\
\hline & No & \\
\hline \multirow{2}{*}{$\begin{array}{l}\text { ¿Has tenido alguna } \\
\text { enfermedad de trans- } \\
\text { misión sexual? }\end{array}$} & Sí & ¿Cómo/ Quién te contagió? \\
\hline & No & \\
\hline
\end{tabular}




\begin{tabular}{|c|c|c|c|}
\hline \multirow{2}{*}{$\begin{array}{l}\text { ¿Has sufrido en el pa- } \\
\text { sado alguna agresión } \\
\text { o abuso sexual por } \\
\text { otra/s persona/s? }\end{array}$} & Sí & \multicolumn{2}{|c|}{$\begin{array}{l}\text { ¿Qué ocurrió? ¿Quién lo hizo? ¿Con qué frecuen- } \\
\text { cia? ¿Durante cuando tiempo? }\end{array}$} \\
\hline & No & & \\
\hline \multirow{3}{*}{$\begin{array}{l}\text { ¿Alguna vez te ha } \\
\text { humillado por haber } \\
\text { llevado la iniciativa } \\
\text { sexual? }\end{array}$} & Sí & \multicolumn{2}{|l|}{ ¿Qué ocurrió? } \\
\hline & No & & \\
\hline & No & & \\
\hline \multirow{11}{*}{$\begin{array}{l}\text { ¿Te has visto algu- } \\
\text { na vez obligada a } \\
\text { mantener relaciones } \\
\text { sexuales con tu pare- } \\
\text { ja o a realizar alguna } \\
\text { conducta sexual que } \\
\text { no desearas? }\end{array}$} & \multirow{11}{*}{ Sí } & \multirow{11}{*}{$\begin{array}{l}\text { ¿Qué tipo de } \\
\text { conductas } \\
\text { sexuales? }\end{array}$} & Tocamientos \\
\hline & & & Masturbación \\
\hline & & & Sexo oral \\
\hline & & & Penetración vaginal \\
\hline & & & Penetración anal \\
\hline & & & Introducción de objetos \\
\hline & & & Visualización de pornografía \\
\hline & & & Sexo con otras personas \\
\hline & & & $\begin{array}{l}\text { Usar ropa u objetos con fines } \\
\text { sexuales }\end{array}$ \\
\hline & & & Exhibicionismo \\
\hline & & & Otros \\
\hline
\end{tabular}

(Continúa) 
Apéndice 2. Entrevista Semiestructurada para la Exploración de la Violencia Sexual sobre las Mujeres en la Relación de Pareja (continuación).

\begin{tabular}{|c|c|}
\hline \multirow{6}{*}{$\begin{array}{l}\text { ¿Cómo te ha } \\
\text { obligado? ¿Cómo ha } \\
\text { reaccionado él si te } \\
\text { negabas? }\end{array}$} & $\begin{array}{l}\text { Con fuerza física } \\
\text { (sujeción, apretones, } \\
\text { empujones, etc.). }\end{array}$ \\
\hline & $\begin{array}{l}\text { Con chantajes o amenazas } \\
\text { (ya sean emocionales, } \\
\text { económicas) o } \\
\text { instantemente. }\end{array}$ \\
\hline & Insultos o menosprecios. \\
\hline & Gritos. \\
\hline & $\begin{array}{l}\text { Conducta agresiva } \\
\text { (por ejemplo: golpes a } \\
\text { mobiliario). }\end{array}$ \\
\hline & Otros. \\
\hline \multirow{6}{*}{$\begin{array}{l}\text { ¿Cómo has } \\
\text { reaccionado tú? }\end{array}$} & $\begin{array}{l}\text { Te has negado } \\
\text { explícitamente. }\end{array}$ \\
\hline & Te has quedado pasiva. \\
\hline & Has fingido placer. \\
\hline & Has llorado. \\
\hline & $\begin{array}{l}\text { Has participado de forma } \\
\text { activa. }\end{array}$ \\
\hline & Otros. \\
\hline \multicolumn{2}{|l|}{$\begin{array}{l}\text { ¿Con qué frecuencia } \\
\text { ocurre? }\end{array}$} \\
\hline $\begin{array}{l}\text { ¿Durante cuánto } \\
\text { tiempo se ha dado } \\
\text { esta situación? }\end{array}$ & \\
\hline
\end{tabular}

Otra información

relevante sobre la

violencia sexual 